\title{
Beating-heart mitral valve surgery: Preliminary model and methodology
}

\author{
Stephen W. Downing, MDa \\ William A Herzog, Jr, MD ${ }^{\mathrm{b}}$ \\ Joseph S. McLaughlin, MD \\ Timothy P. Gilbert, MD ${ }^{c, d}$
}

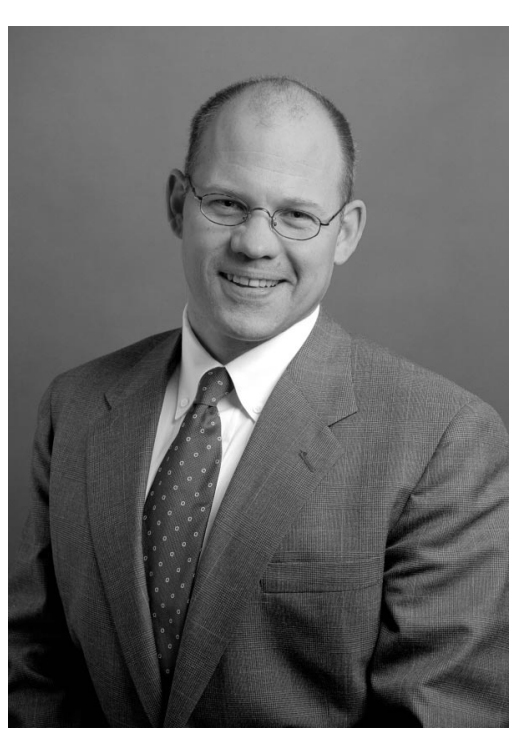

Dr Downing
From the Divisions of Cardiac Surgery, ${ }^{\mathrm{a}}$ Cardiology, ${ }^{\mathrm{b}}$ and Cardiac Anesthesiology ${ }^{\mathrm{c}}$ and the Department of Medicine, ${ }^{\mathrm{d}}$ University of Maryland School of Medicine, Baltimore, Md.

Read at the Eighty-first Annual Meeting of The American Association for Thoracic Surgery, San Diego, Calif, May 6-9, 2001.

Received for publication May 11, 2001; revisions requested July 5, 2001; revisions received Sept 10, 2001; accepted for publication Oct 31, 2001.

Address for reprints: Stephen W. Downing, MD, Cardiac Surgery, Suite N4W94, University of Maryland Medical Center 22 S Greene St, Baltimore, MD 21201 (E-mail: sdowning@smail.umaryland.edu).

J Thorac Cardiovasc Surg 2002;123:1141-6

Copyright (C) 2002 by The American Association for Thoracic Surgery

0022-5223/2002 \$35.00+0 $\quad \mathbf{1 2 / 6 / 1 2 1 6 8 0}$

doi: $10.1067 / \mathrm{mtc} .2002 .121680$
Objective: It is our hypothesis that image-guided mitral valve repair can be performed on the beating heart without cardiopulmonary bypass. As a first phase, we tested the feasibility of suturing the anterior and posterior mitral valve leaflets under image guidance.

Methods: In a water bath model, imaging approaches and suturing techniques were developed. Then, in 6 pigs, the left atrium was cannulated with a custom-made $15-\mathrm{mm}$ valved port through a left thoracotomy. Atrial pressure was elevated by shunting of arterial blood to minimize air induction. A multiplane transesophageal echocardiographic probe was evaluated in the intraesophageal and epicardial positions. With a commercial endoscopic suturing device, sutures were placed through the anterior and posterior mitral leaflets under echocardiographic guidance. The animals were killed, and suture accuracy was evaluated by measuring the distance from the intended target areas on the anterior and posterior mitral leaflets. Air induction was monitored by echocardiography and graded as minimal to severe.

Results: There were no cases of hemodynamic instability or significant arrhythmia. The most effective imaging plane was a short-axis view that used the transesophageal echocardiographic probe epicardially at the heart base. Air induction was minimal in 2 animals, mild in 3, and moderate in 1 . Sutures were successfully placed 9 of 12 times (mean error $0.8 \pm 0.5 \mathrm{~cm}$ ).

Conclusions: With these methods, off-pump, image-guided suturing of the beatingheart mitral valve was possible. This model may be a useful starting point for developing off-pump mitral valve repair procedures.

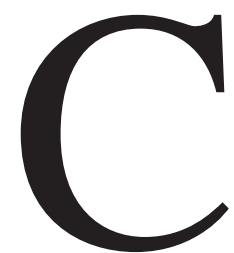

ardiac valve repair and replacement have become progressively less invasive. ${ }^{1}$ The newer small-incision approaches increase patient satisfaction and may reduce postoperative morbidity. ${ }^{2-5}$ They do not, however, eliminate the cost and morbidity associated with cardiopulmonary bypass, cardiac arrest, or cardioplegia. ${ }^{6,7}$

Early cardiac surgical procedures, such as atrial septal defect (ASD) closure and mitral commissurotomy, were first performed on the beating heart. ${ }^{8-14}$ Because these operations were undertaken blindly, accuracy was limited and the results were often suboptimal. The advent of cardiopulmonary bypass allowed direct visualization of the surgical target, and this soon became the standard method of operation. Our working hypothesis is that if conventional visualization (direct vision or endoscopic camera imaging) could be satisfactorily replaced with another modality, it might be possible to eliminate cardiopulmonary bypass from many traditionally open cardiac surgical procedures. Our long-term goal is to develop the imaging systems, instrumentation, and techniques necessary to perform closed-chest beating-heart cardiac 


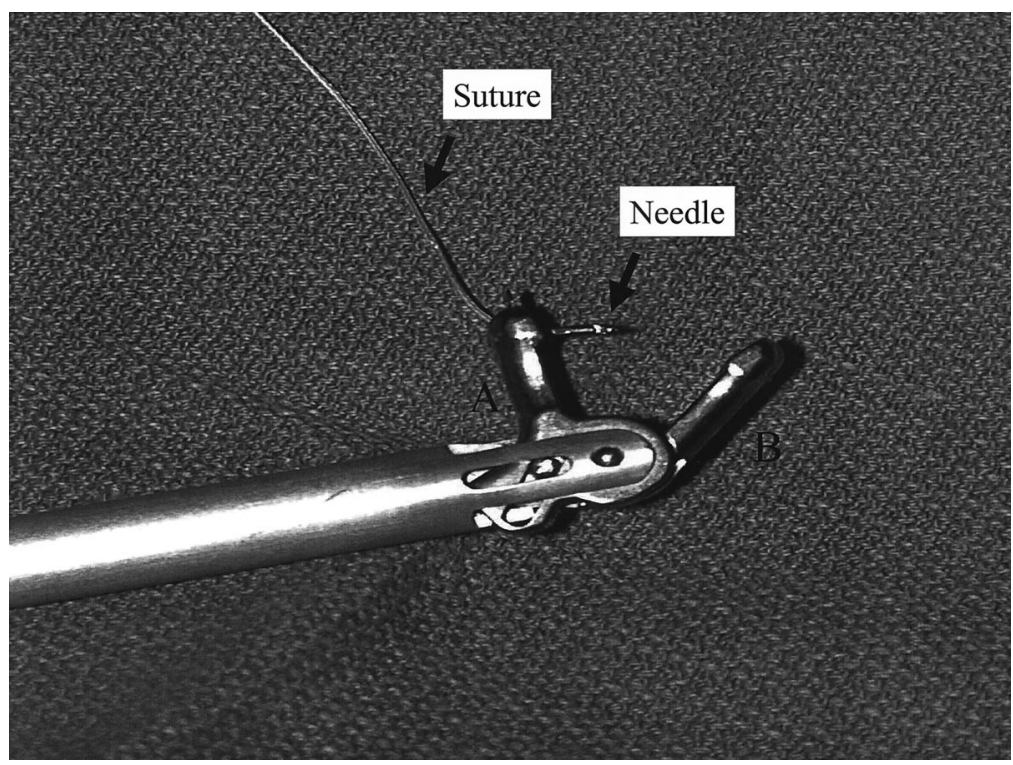

Figure 1. Endoscopic suturing device (CardioStitch; United States Surgical Corporation, Norwalk, Conn). Target tissue is grasped between arms $\boldsymbol{A}$ and $\boldsymbol{B}$. Lever switches grasp of needle between arms. Needle is always controlled by arm. This reduces risk of embolization and does not require second hand to grasp needle.

surgical procedures, such as mitral valve repair, arrhythmia ablation, and atrial septal defect closure.

The hypothesis for this study was that the mitral valve can undergo safe and accurate surgical manipulation under echocardiographic guidance without the use of cardiopulmonary support. In the course of this evaluation, the most appropriate echocardiographic windows and probe positions were identified, and the strengths and limitations of 2dimensional echocardiography as the primary visualizing modality were demonstrated. Additionally, the utility of our first generation of cardiac access ports was tested.

\section{Methods}

\section{Imaging System}

All imaging was performed with an Omniplane transesophageal echocardiographic (TEE) probe machine (Omniplane; Phillips Medical Systems, Elmsford, NY) displaying images on a Sonos 1500 monitor (Phillips Medical Systems).

\section{Basic Techniques}

An ASD model in a water bath was used to develop the initial surgical and imaging techniques. The $\operatorname{ASD}(n=6)$ was created by excising the septum of slaughterhouse porcine hearts. The echocardiographic probe was placed in multiple positions above and below the defect, and a series of basic tasks was attempted with only the echocardiographic images used for guidance. These tasks included the following:

1. The surgeon (S.W.D.) grasped targeted areas on the midportion and apices of the ASD with an endoscopic grasper (Genzyme Corporation, Cambridge, Mass).
2. The surgeon sewed the defect closed with a 4-0 Prolene suture (Ethicon, Inc, Somerville, NJ) on an SH needle with an endoscopic needle driver (Genzyme Corporation) and an endoscopic grasper (Genzyme Corporation)

3. The surgeon sewed the defect closed with an endoscopic suturing device as shown in Figure 1 (CardioStitch; United States Surgical Corporation, Norwalk, Conn).

\section{Mitral Valve Surgery}

Six 50- to $60-\mathrm{kg}$ pigs were placed under general anesthesia and intubated. Femoral artery pressure and cardiac rhythm were monitored. A right thoracotomy was performed, and a purse-string suture was placed in the left atrial appendage. A specially constructed 15-mm diameter valved port (Figure 2) was inserted through the appendage after the administration of 5000 units of heparin. To minimize blood loss and air entry, the port had a snugly fitting rubber inlet aperture that the instruments passed through and a bileaflet flap valve to seal the port when the instruments were out. An inlet below the flap valve received blood from a small-caliber catheter in the femoral artery. The blood flow from the higher-pressure femoral artery was intended to maintain an elevated pressure in the left atrium throughout the respiratory cycle (and in periods of relative hypovolemia) to reduce the chance of air entry.

To compare for utility the various possible echocardiographic probe positions and windows, a repeated series of maneuvers was performed (grasping and release of each commissure, grasping and release of the midportion of the anterior and posterior leaflets). For each of these maneuvers, the surgical operator (S.W.D.) rated his ability to see and understand the anatomic targets and his ability to comprehend the operative field in a surgically useful manner on an arbitrary scale ( 0 , cannot comprehend; 1 , can comprehend with 


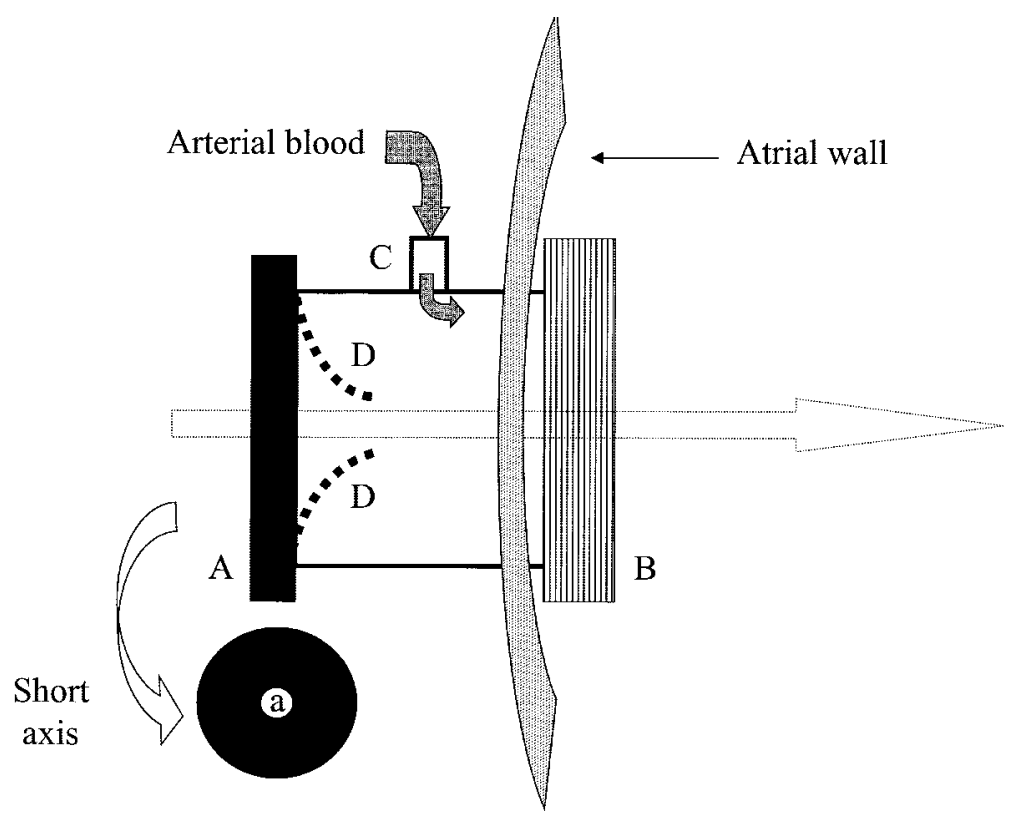

Figure 2. Schematic drawing of cardiac access port. $A$, Rubber inlet seal fits snugly around instrument passed through opening a. $B$, Flange to prevent outward migration. Arterial blood is shunted from femoral artery into inlet value $C$ to elevate intra-arterial pressure. Arrow $D$ demonstrates instrument path into heart.

moderate effort; 2 , can comprehend with minimal effort). The true TEE probe position was abandoned because of poor imaging resulting from the orientation of the pig atrium in relation to the esophagus. The echocardiographic probe was used in an epicardial location on the left atrium to mimic the midesophageal probe positions and along the inferior surface of the ventricle in the transverse sinus to mimic the transgastric views. With these two probe positions, the epicardial analogs of the following conventional TEE views were evaluated: the midesophageal 4-chamber, 2-chamber, and long-axis views and the transgastric 2-chamber, long-axis, and short-axis views.

After selection of the best echocardiographic probe locations and imaging planes, the suturing device was placed into the atrium, and the placement of a suture was attempted through the midportion of the anterior and posterior leaflets to mimic an Alfieri-type repair. The animals were killed, the suture positions were noted, and the distances from the ideal midpoint location were measured.

The echocardiographic images were recorded on videotape during the valve manipulation and suturing. The amount of air entering the heart was graded by a single reviewer (T.P.G.) on an arbitrary scale from minimal (rare air bubbles) to severe (chamber filled with bubbles). Each animal was scored according to the worst grade observed during the surgical period.

All animals received humane care in compliance with the Guide for the Care and Use of Laboratory Animals prepared by the institute of Laboratory Animal Resources, National Research Council, and published by the National Academy press, revised 1996. All protocols were approved by the animal care committee of the University of Maryland.

\section{Results}

In the water bath, the simple maneuvers of grasping the ASD edge with the grasper and endoscopic suture device were performed within $5 \mathrm{~mm}$ of the target area at all times. The endoscopic suturing device successfully passed all sutures through the defect edges. Conventional suturing with a needle driver and grasper was not possibly because of the inability to reliably regrasp the needle after it was passed through the tissue.

In the porcine model, the only echocardiographic probe position that scored greater than 1 for both anatomic and surgical comprehension was the transgastric-analog basilar short-axis position. This fishmouth view of the mitral valve allowed excellent $\mathrm{x}$-axis (left to right) and $\mathrm{y}$-axis (anterior to posterior) orientations. Information regarding the $\mathrm{z}$-axis (long axis of the ventricle) was obtained by advancing the echocardiographic probe toward the apex and then back again along this axis.

The sutures were placed as desired on the valve 9 of 12 attempts ( 1 attempt per leaflet). Three of 12 sutures snared in the chordae. The mean distance from the ideal position at the midportion of the leaflet was $0.8 \pm 0.5 \mathrm{~cm}$ (Figure 3).

There were no cases of significant arrhythmia or hemodynamic instability observed during the procedures. Air induction was minimal in 2 animals, mild in 3, and moderate in 1 . 


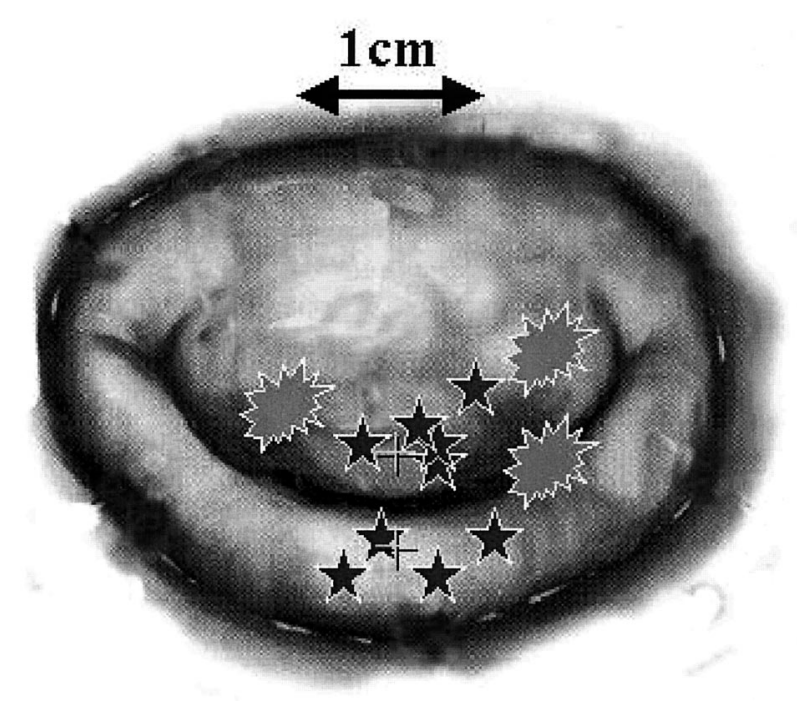

Figure 3. Composite drawing of mitral valve viewed from left atrium. Stars mark approximate placement of individual sutures. Bursts indicate sutures that were snared in chordae or were placed into subvalvular apparatus.

\section{Discussion}

The concept of image-guided intracardiac surgery is an old one. In the 1920s, Allen and Graham ${ }^{13}$ and Wilson ${ }^{14}$ independently described the use of a cardioscope to guide intracardiac surgery. The visualization was poor, however, and the idea did not develop further. Modern technology may allow a reexploration of this approach. We believe that it is feasible that in the near future such procedures as ASD closure, ablation of atrial fibrillation, and mitral valve repair will be performed on the closed-chested, beating heart by robotic devices inserted into the heart and guided totally by echocardiography or magnetic resonance imaging.

Access to the heart could be obtained through a port placed into the left atrium through an anterolateral minithoracotomy, or preferably by thoracoscopic techniques. The cardiac access port that we fabricated worked adequately, allowing safe cardiac access without significant air entry and blood loss. In early port testing, air induction generally occurred when the animal was hypovolemic and the atrial pressure was low. It was hypothesized that shunting blood into the left atrium from the femoral artery would keep left atrial pressure elevated under most conditions, thus reducing the risk of air entry. This concept has not been separately tested. In addition to the design of the port, air entry also depends on the instruments. The adapted laparoscopic instruments that we used carried some air into the heart in their hollow shafts and also along irregularities on the instrument surfaces. Instruments designed specifically for closed-chest cardiac surgery will need to avoid these features. The second generation of the cardiac port, currently under development, incorporates two trileaflet valves and has a tighter fit around the instruments, which we hope will further reduce air entry.

Among the systems currently available for image guidance, echocardiography is the most appealing because of its real-time nature (unlike conventional computed tomography and magnetic resonance imaging, which require time to construct each individual image frame), lack of ionizing radiation or magnetic fields, and relatively low cost. Echocardiographic imaging has been used to visualize and guide many procedures, such as cannula placement in less-invasive cardiac surgical procedures, transseptal puncture for left heart catheterization, device closure of ASD, endomyocardial biopsy, and guidance of ablation catheters for arrhythmia treatment. ${ }^{15-24}$ Despite this widespread use, the best techniques for the use of echocardiography to guide procedures have not been well described. In our experience, a TEE probe in the epicardial position was effective. The epicardial position generated images superior to those generated by the intraesophageal location in this model and allowed more flexibility in choosing imaging windows. A TEE probe may be superior to other probe designs (such as those used for epiaortic scanning), because the small probe head and flexible probe neck allow placement in such diverse locations as the transverse sinus and into the right atrium proper. The most useful images were obtained with the probe in the transverse sinus with a basilar short-axis window. This provided a full fishmouth view of the valve, familiar to surgeons who are accustomed to viewing the mitral valve from the left atrium. To obtain information about structures above or below the cross-section being viewed, the probe must be advanced toward the apex or base. Alternatively, the imaging plane can be rotated (without moving the probe) to a long-axis view; however, this view may be incomplete and confusing if the shaft of the device is not completely parallel to the imaging plane, which it rarely is.

The major shortcoming of conventional TEE is the frequent need to use more than one transducer position to comprehend the whole 3-dimensional anatomy. A true 3dimensional image would be much simpler and more intuitively useful. There are commercially available systems that can generate moving, 3-dimensional echocardiographic images (TomTec GmbH, Munich). However, several seconds are required for the construction of each individual image set, making such images impractical as a surgical guide. Real-time 3-dimensional echocardiography will be a significant advancement, and prototype devices are currently being evaluated (Volumetrics Corporation, Durham, $\mathrm{NC})$.

The Alfieri approach ${ }^{25}$ was chosen for this study solely because of its simplicity. This type of repair may have a role as the sole therapy in certain types of mitral regurgitation, 
but the indications for this limited approach will need to be defined and the long-term results will need to be demonstrated. ${ }^{26-28}$ We initially tied the sutures after placement in the valve with a laparoscopic knot pusher. This was stopped after the third animal, when we tore the leaflet (which are very thin in young pigs), so that we could tell what damage may have occurred from the suturing device and what occurred during tying. For clinical use we expect a securing device or suture weld would be more reliable than hand sewing.

For beating-heart mitral valve surgery to be widely applied, a number of complementary surgical maneuvers will need to be developed. Resectional procedures on the beating heart would be difficult because of the risk of embolization. However, it may be possible to circumvent this problem with modified versions of standard techniques. For example, quadrangular resection could be replaced by a combination of leaflet plication and chordal replacement. Along that line, we are currently developing the instrumentation to perform sewn and stapled mitral annuloplasties, which would be similar to standard open mitral ring annuloplasties.

\section{Conclusions}

Rudimentary beating-heart mitral valve surgery is possible. Two-dimensional echocardiography can be used to guide surgical procedures by adhering to certain principles. Through the use of our paradigm, with improved instrumentation and imaging systems, the performance of beatingheart mitral valve repairs may be possible in the near future.

\section{References}

1. Cosgrove DM, Sabik JF, Navia JL. Minimally invasive valve operations. Ann Thorac Surg. 1998;65:1535-8.

2. Slone RM, Pilgram TK, Gierada DS, Sagel SS, Glazer HS, Yusen RD, et al. Lung volume reduction surgery: comparison of preoperative radiologic features and clinical outcome. Radiology. 1997;204:685-93.

3. Cohn LH, Adams DH, Couper GS, Bichell DP, Rosborough DM, Sears SP, et al. Minimally invasive cardiac valve surgery improves patient satisfaction while reducing costs of cardiac valve replacement and repair. Ann Surg. 1997;226:421-6.

4. Sugi K, Kaneda Y, Murakami T, Esato K. The outcome of volume reduction surgery according to the underlying type of emphysema. Surg Today. 2001;31:580-5.

5. Gundry SR, Shattuck OH, Razzouk AJ, del Rio MJ, Sardari FF, Bailey LL. Facile minimally invasive cardiac surgery via ministernotomy. Ann Thorac Surg. 1998;65:1100-4.

6. Downing SW, Edmunds LH. Release of vasoactive substances during cardiopulmonary bypass. Ann Thorac Surg. 1992;54:1236-43.

7. Edmunds LH. Why cardiopulmonary bypass makes patients sick: strategies to control the blood-synthetic surface interface. Adv Card Surg. 1995;6:131-67.

8. Cutler EC, Levine SA. Cardiotomy and valvulotomy for mitral stenosis. Bost Med Surg J. 1923;188:1022-7.

9. Watins E Jr, Pomeranz AA, Goldsmith EI, Gross RE. Experimental closure of atrial septal defects: technique of an "atrial well" operation. Surg Forum. 1952;3:247-52.

10. Watkins E Jr, Gross RE. Experiences with surgical repair of atrial septal defects. J Thorac Cardiovasc Surg. 1955;30:469-91.

11. Gross RE, Pomeranz AA, Watkins E Jr, Goldsmith EI. Surgical closure of defects of the interauricular septum by use of an atrial well. N Engl J Med. 1952;247:455-60.
12. Gross RE, Watkins E Jr. Surgical closure of atrial septal defects. Arch Surg. 1953;67:670-81.

13. Allen DS, Graham EA. Intracardiac surgery-a new method. JAMA. 1922;79:1028-30.

14. Wilson WG. Studies in experimental mitral obstruction in relation to the surgical treatment of mitral stenosis. Br J Surg. 1930;18:259-74.

15. Applebaum RM, Colvin SB, Galloway AC, Ribakove GH, Grossi EA, Tunick PA, et al. The role of transesophageal echocardiography during port-access minimally invasive cardiac surgery: a new challenge for the echocardiographer. Echocardiography. 1999;16:595-602.

16. Schulze CJ, Wildhirt SM, Boehm DH, Weigand C, Kornberg A, Reichenspurner $\mathrm{H}$, et al. Continuous transesophageal echocardiographic (TEE) monitoring during port-access cardiac surgery. Heart Surg Forum. 1999;2:54-9.

17. de Moor M, Pribut H, Hugo-Hamman C. The switch to balloon atrial septostomy with echocardiographic guidance - a severe complication. S Afr Med J. 1996;86 Suppl 2:C97-C9.

18. Fisher WG, Pelini MA, Bacon ME. Adjunctive intracardiac echocardiography to guide slow pathway ablation in human atrioventricular nodal reentrant tachycardia: anatomic insights. Circulation. 1997;96: 3021-9.

19. Kawauchi M, Gundry SR, Boucek MM, de Begona JA, Vigesaa R, Bailey LL. Real-time monitoring of the endomyocardial biopsy site with pediatric transesophageal echocardiography. J Heart Lung Transplant. 1992;11(2 Pt 1):306-10.

20. Ren JF, Schwartzman D, Callans DJ, Brode SE, Gottlieb CD, Marchlinski FE. Intracardiac echocardiography (9 MHZ) in humans: methods, imaging views and clinical utility. Ultrasound Med Biol. 1999; 25:1077-86.

21. Wilkens H, Demertzis S, Konig J, Leitnaker CK, Schafers HJ, Sybrecht GW. Lung volume reduction surgery versus conservative treatment in severe emphysema. Eur Respir J. 2000;16:1043-9.

22. Marchlinski FE, Ren JF, Schwartzman D, Callans DJ, Gottlieb CD. Accuracy of fluoroscopic localization of the crista terminalis documented by intracardiac echocardiography. J Interv Card Electrophysiol. 2000;4:415-21.

23. Chan KC, Godman MJ, Walsh K, Wilson N, Redington A, Gibbs JL. Transcatheter closure of atrial septal defect and interatrial communications with a new self expanding nitinol double disc device (Amplatzer septal occluder): multicentre UK experience. Heart. 1999;82: 300-6.

24. Roithinger FX, Steiner PR, Goseki Y, Liese KS, Scholtz DB, Sippensgroenewegen A, et al. Low-power radiofrequency application and intracardiac echocardiography for creation of continuous left atrial linear lesions. J Cardiovasc Electrophysiol. 1999;10:680-91.

25. Alfieri O, Maisano F. An effective technique to correct anterior mitral leaflet prolapse. J Card Surg. 1999;14:468-70.

26. Maisano F, Schreuder JJ, Oppizzi M, Fiorani B, Fino C, Alfieri O. The double-orifice technique as a standardized approach to treat mitral regurgitation due to severe myxomatous disease: surgical technique. Eur J Cardiothorac Surg. 2000;17:201-5.

27. Maisano F, Torracca L, Oppizzi M, Stefano PL, D'Addario G, La Canna G, et al. The edge-to-edge technique: a simplified method to correct mitral insufficiency. Eur J Cardiothorac Surg. 1998;13:240-5.

28. Umana JP, Salehizadeh B, DeRose JJ Jr, Nahar T, Lotvin A, Homma $\mathrm{S}$, et al. "Bow-tie" mitral valve repair: an adjuvant technique for ischemic mitral regurgitation. Ann Thorac Surg. 1998;66:1640-6.

\section{Discussion}

Dr James L. Cox (Washington, DC). I congratulate Downing and colleagues on a unique and remarkable approach to a difficult problem. Dr Downing, would you expand a little on your comments relating to chordal replacement? Are you actually putting polytetrafluoroethylene sutures in and attaching them to the papillary muscle and then to the leaflet, as we would in an open procedure?

Dr Downing. We can do that in a very crude fashion, but the tying part of it is not too good. We are really focusing on the imaging now, because echocardiographic imaging is just not good 
enough to extend this modality to human beings. We are focusing more on the best use and accuracy of the 2-dimensional and 3-dimensional echocardiographic systems.

Dr Cox. When you go from putting an Alfieri stitch in to changing over to an annuloplasty, even just tucking the corners, do you have to change the plane of the echocardiography at that point? Do you still use the same plane, or do you have to change the echocardiographic probe position?

Dr Downing. You use the same general echocardiographic probe position. The Omniplane probe lets you switch the imaging plane electronically, and then you add some small supplemental position adjustments.

Dr Cox. Do you open the pericardium?

Dr Downing. Yes.

Dr Cox. Okay. So the pericardium is open and left open, and this chamber is put against the right atrium?

Dr Downing. Into the right atrium. We just make a purse-string suture and place the port right into the left atrium proper, and then that is your access into the heart.

Dr Cox. Well, it is a unique idea, and I congratulate you on it.

Dr Alain F. Carpentier (Paris, France). I rise just to say that for those of us who know a little bit about mitral valve reconstruction, this approach seems to be totally unrealistic, if not quite dangerous. It is unfortunate that you were not able to show us the hearts of these pigs after such an operation, the more so because if this operation works it will be used in elderly people with fibroelastic deficiency with a P2 prolapse. We know that the tissues are extremely fragile and the suturing of the anterior leaflet to the posterior leaflet, which is anyway only a palliative operation, is already difficult with an open procedure. It would have been more convincing if you had shown to us the specimens of the heart after having performed such an operation. That's number one.

Number two, you were talking about being able to perform annuloplasty. It is hard for me to understand how you can suture anything with your device that requires an edge to be placed into position.

Number three, with respect to chordal replacement, we all know that the difficulty for chordal replacement or transfer is to adjust the length. How can you foresee that this is possible on a beating heart, given that 2- or 3-mm difference can lead to a residual leak?

I do not mean to be unkind, but just to stimulate the discussion. I congratulate you on the efforts you are making to develop this technology.
Dr Downing. Your comments are certainly appreciated and expected. Perhaps I emphasized the mitral valve just a bit too much. Our goal is to develop a way to operate inside of the beating heart safely through ports. When you can do that, you can close atrial septal defects. You can take a robot and do your maze procedure with a cryoprobe or a radiofrequency probe. You can take biopsy specimens and do other trivial procedures. You can do closed commissurotomies with a robot that the cardiologists are doing with a balloon, and you can do a better job for your patient.

I apologize again if I emphasized the mitral valve too much. This is just the model that we used to learn how to image and to learn how to sew. There is no end, I think, to the possible ways to repair the mitral valve once you have the basic methods down. We may be able to do stapled annuloplasties or sutured annuloplasties, or sew in mitral rings in with machines that are on the end of a robotic arms.

So again, I am sorry if I did not communicate my goals and what we are trying to do clearly enough. Our intent is not to come up with a mitral valve repair particularly. It is to learn how to operate inside the beating heart with imaging technology as the sole visualization.

Dr Pedro J. Del Nido (Boston, Mass). My question relates to your choice of imaging modality. Magnetic resonance imaging clearly will give you a better image, despite its difficulties because of magnetic fields. Have you considered magnetic resonance imaging, particularly now that there are open magnets available that will give you a real-time and potentially 3 -dimensional image?

Dr Downing. We chose this imaging modality because echocardiography is the cheapest of the choices and has the most potential for real-time 3-dimensional imaging. The problem with 3-dimensional magnetic resonance imaging is that techniques currently require off-line reconstruction. So you image for a sequence, and then the system reconstructs the picture for you. If you have a needle in your hand and are trying to approach the anulus or trying to close a septal defect, you cannot wait for the image to generate before you move your hand again.

With respect to computed tomographic systems, you have to get a volume of data. That means that the table is going to be moving back and forth or the ring will be moving back and forth. So that is not going to work yet. But we actually are working with one of the computed tomographic imaging companies to try to do this. The magnetic fields required for magnetic resonance imaging are currently not compatible with operating room equipment. 